\title{
Screening-detected desmoid tumor of the breast: findings at conventional imaging and digital breast tomosynthesis
}

\author{
Tatjana Samardzic', Jon Lømo ${ }^{2}$ and Per Skaane ${ }^{3}$
}

\begin{abstract}
Desmoid tumor of the breast is a rare benign entity that usually is mistaken for carcinoma clinically and radiologically. We report two cases of desmoid tumor of the breast detected by mammography screening using digital breast tomosynthesis (DBT). The larger tumor was detected at both full-field digital mammography (FFDM) and DBT. The smaller desmoid tumor, however, was identified only at tomosynthesis. Mammographic and ultrasonographic findings at diagnostic workup were consistent with carcinoma of the breast. Preoperative needle biopsies could not conclusively diagnose the lesions. Both patients underwent excisional biopsy and histopathology revealed fibromatosis of the desmoid type.
\end{abstract}

\section{Keywords}

Mammography screening, digital breast tomosynthesis, desmoid tumor, fibromatosis

Date received: 30 November 2017; accepted: 8 December 2017

\section{Introduction}

Desmoid tumor (synonym: desmoid-type fibromatosis) is a benign lesion of the connective tissue with a tendency to infiltrate adjacent tissue but without metastatic potential. Development in the breast is very rare (1-4). Desmoid of the breast represents a diagnostic challenge because the lesions mimic breast carcinoma radiologically (5-7).

We report two cases of desmoid tumor detected at breast cancer screening using digital breast tomosynthesis (DBT). The smaller lesion was only identified at tomosynthesis. DBT is a new promising mammographic technique that solves many of the limitations of conventional mammography. A careful review of the literature did not reveal earlier reports of desmoid tumor of the breast evaluated by tomosynthesis.

\section{Case reports}

\section{Case I}

A 62-year-old woman was recalled from screening because of a spiculated mass in the left breast. She underwent breast reduction mammoplasty since her last screening examination two years earlier.
There was no history of breast cancer in her family. On clinical exam at assessment, there were visible scars after mammoplasty, but no palpable mass.

A high-density spiculated mass, measuring $15 \mathrm{~mm}$ and located in the lower medial portion of the left breast close to the thoracic wall, was highly suggestive of malignancy at full-field digital mammography (FFDM) as well as DBT. The desmoplastic reaction (spiculations) was more obvious at DBT than at FFDM (Fig. 1a and b). Ultrasonography revealed a 13-mm hypoechoic irregular mass close to the pectoral muscle. The ultrasound finding was consistent with infiltration of the pectoral muscle. The mass was consistent with a malignant tumor. Ultrasonographic examination of the axilla was negative.

\footnotetext{
'Department of Radiology, Oslo University Hospital, Oslo, Norway ${ }^{2}$ Department of Pathology, Oslo University Hospital, Oslo, Norway ${ }^{3}$ Department of Radiology, Oslo University Hospital, University of Oslo, Oslo, Norway
}

\section{Corresponding author:}

Tatjana Samardzic, Oslo University Hospital, Department of Radiology, Breast Imaging Center, Ullernchauseen 64-66, 0379 Oslo, Norway. Email: tatsam@ous-hf.no 
The patient underwent ultrasound-guided fine needle aspiration cytology (FNAC) which showed atypical cells suspicious of infiltrating ductal carcinoma. Core needle biopsy (CNB) was recommended and US-guided CNB revealed fibrotic tissue but no carcinoma.

Due to discordance of the imaging and pathological findings, excisional biopsy was carried out. Histopathology showed fibromatosis of desmoid tumor type with infiltration of the pectoral muscle (Fig. 1c and d). To ensure complete resection and prevent recurrence, a re-resection was performed but no residual tumor was found. Two screening rounds later (the second with DBT), there were no signs of local recurrence.

\section{Case 2}

A 65-year-old woman was recalled from mammography screening because of a distortion in the upper medial portion of the right breast not seen at $2 \mathrm{D}$ (reconstructed synthetic 2D images) (Fig. 2a and c) but detected at tomosynthesis (Fig. 2b and d). There was no history of breast cancer. Clinical examination at work-up was normal without any palpable mass.

At assessment, conventional mammography including fine-focus magnification views and cone-down views were performed. The small spiculated mass could not be confirmed at conventional supplemental views but was again only confirmed at DBT. Ultrasound demonstrated a 6-mm irregular hypoechoic (a)

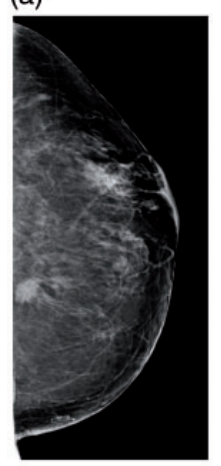

(b)

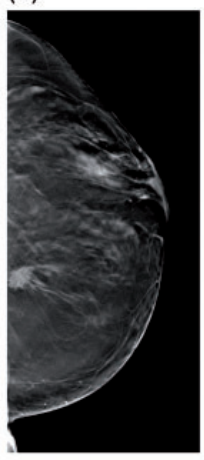

(c)

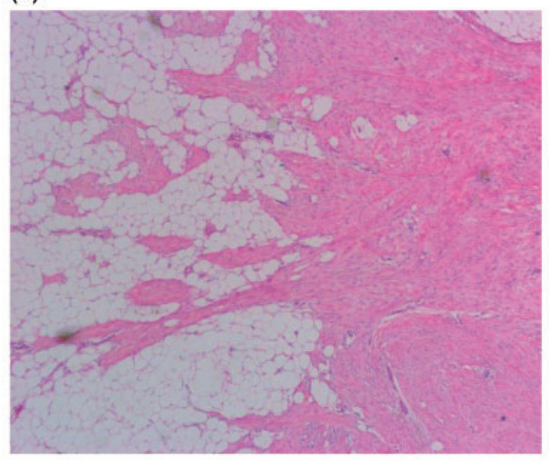

(d)

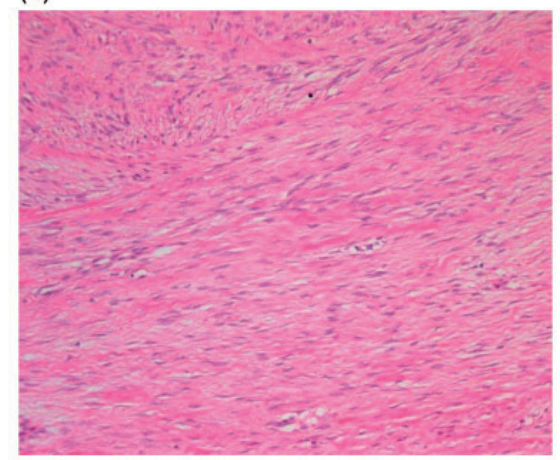

Fig. I. (a) FFDM left breast (craniocaudal [CC] projection) shows a high-density spiculated mass in the medial-posterior fatty part of the breast. (b) DBT in the same projection confirms the FFDM findings. The spiculations are more clearly presented at DBT, but no additional information is given. Histopathology of the surgical specimen of case I (hematoxylin and eosin stain; $40 \times$ [left panel] and $200 \times$ [right panel] magnification). (c) The irregular margins of the lesion with infiltrative processes in the fat tissue, which mimic an infiltrating carcinoma. (d) On higher magnification, the long sweeping bundles of bland, cytologically benign spindle cells can be seen. Normal breast glands may be trapped within the lesion. Immunostains were negative for cytokeratins and p63, thereby excluding a carcinoma diagnosis.

(a)

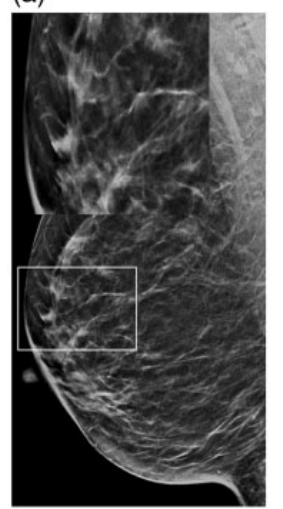

(b)

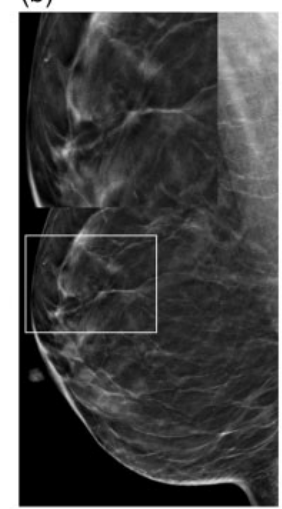

(c)

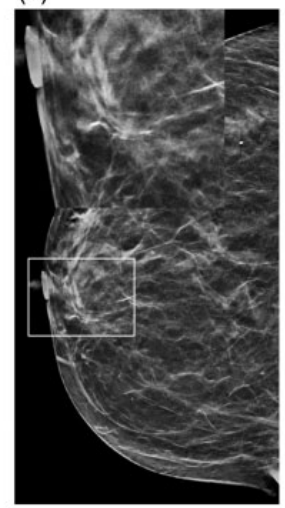

(d)

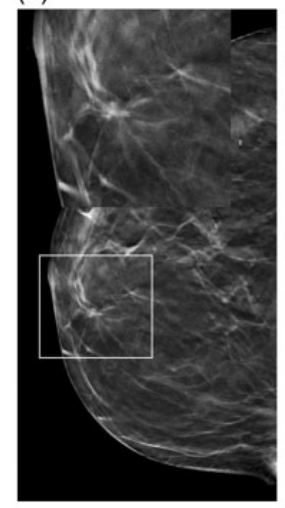

(e)

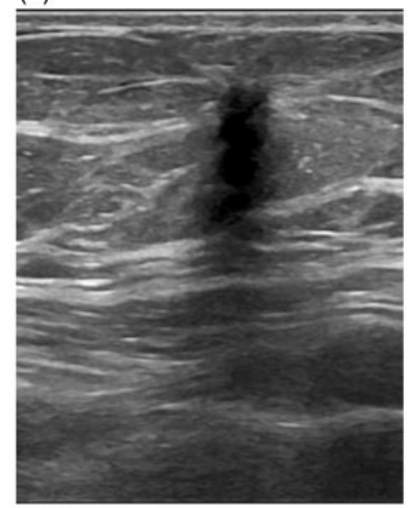

Fig. 2. (a) Synthetic 2D mammogram, Mediolateral oblique view right breast showing scattered fibroglandular tissue but no sign of malignancy. (b) DBT shows a small non-specific mass. (c) Synthetic 2D CC view demonstrates a suspicious area but the finding is inconclusive. (d) DBT in CC projection clearly demonstrates a small spiculated mass. (e) Ultrasound showing irregular hypoechoic mass with posterior shadowing, typical of carcinoma. 
mass at 2 o'clock with extensive posterior shadowing highly suspicious of malignancy (Fig. 2e). Ultrasound of the axilla was negative.

Ultrasound-guided CNB showed fibrosis but no malignancy. At interdisciplinary meeting, a core needle biopsy was recommended, which again showed only benign tissue. The patient then underwent excisional biopsy due to discordance of imaging and CNB findings. Histopathology showed fibromatosis of desmoid type.

No further intervention was recommended. There was no sign of local recurrence at screening mammography two years later.

\section{Discussion}

Desmoid tumor or desmoid-type fibromatosis is an entity of unknown etiology, defined by the World Health Organization (WHO) as "clonal fibroblastic proliferation arising in the deep soft tissues, with a tendency to infiltrate local tissues and locally recur, but an inability to metastasize" $(1,8,9)$. Desmoid-type fibromatosis usually presents in the age range of 15-60 years and is more than twice as common in women (8). Desmoid may occur in any anatomical region and is usually classified as intra- and extra-abdominal fibromatosis of desmoid type $(2,8)$

There is a wide range of clinical manifestations depending on localization. Patients may complain of pain, a palpable mass, or different symptoms related to involvement of adjacent organs (8).

Histopathologically, desmoid-type fibromatosis is an infiltrative, locally aggressive proliferation of fibroblasts and myofibroblasts. These spindle cells form long, uniform fascicles that extend into the surrounding tissue (Fig. 1d and e), resulting in entrapment of normal breast tissue within the lesion. There can be mitoses, but there is no nuclear atypia. The most important differential diagnosis includes spindle cell carcinoma of fibromatosis-type, traumatic scarring and nodular fasciitis, and rarely fibrosarcoma (10-12).

Desmoid tumor of the breast is a rare entity accounting for only $0.2 \%$ of all breast masses. It is also referred to as mammary fibromatosis or aggressive fibromatosis $(2,3,13,14)$. The desmoid tumor of the breast can arise in the pectoral muscle or in the breast tissue $(6,15)$, and may present clinically as a painless, firm, usually mobile mass occasionally fixed to the pectoral muscle or skin. Nipple discharge or palpable lymphadenopathy is not present $(3-6,13)$. The patients in our study did not have any clinical findings: the lesion in case 2 was very small and detected only at tomosynthesis, and the lesion in case 1 was located deep in the breast. Extraabdominal desmoid tumor has also been reported in the axilla (16).
Breast surgery, including excisional biopsy, lumpectomy, mastectomy, breast reduction and augmentation, reconstruction, and breast implants, has been reported in women presenting with desmoid tumor $(4-6,14)$, suggesting that it can be induced by trauma. Our patient case 1 underwent breast reduction surgery two years before the diagnosis.

Therapy for desmoid tumor in the breast is surgical, with wide excision and clear margins. Positive margins are associated with higher rates of recurrence $(6,13,15)$.

Imaging findings of desmoid fibromatosis include usually an irregular and spiculated high-density non-calcified mass at mammography $(6,7)$. Ultrasonographic appearance is often a hypoechoic, irregular mass with posterior shadowing and echogenic rim $(6,7)$. Similar to the reports in the literature, the ultrasonographic findings in both our cases were a hypoechoic, irregular mass highly suspicious of malignancy. Rarely, desmoid can be seen as a benign mass with circumscribed borders and posterior acoustic enhancement $(6,13)$. On magnetic resonance imaging (MRI), desmoid tumor has been described as an isointense mass on T1-weighted images, and as a lower or higher intensity lesion on T2-weighted images. Contrast-enhanced MRI may show heterogeneous enhancement atypical for breast cancer, and even a more rapid enhancement pattern has been seen in a case of recurring desmoid tumor $(5,13)$. MRI may have a role in preoperative planning, in cases of suspected involvement of thoracic wall, or in cases of recurrent disease $(6,13)$.

DBT is a new, promising, mammographic technique, which has the potential to improve the two inherent limitations of conventional mammography: the low sensitivity in women with dense breast parenchyma caused by a "masking effect" of overlying breast parenchyma, and the low specificity caused by summation of normal breast parenchyma (17). Screening with FFDM plus DBT significantly increases the cancer detection rate compared with FFDM alone (18). Of much importance is the experience that DBT may detect small invasive cancers manifesting as spiculated mass or architectural distortion that are occult on conventional mammography $(19,20)$. The radiation problem of DBT plus FFDM (i.e. a double exposure) might be solved by using reconstructed 2D images (synthesized mammograms), which have been shown to have comparable diagnostic performance compared with FFDM images (21). The larger desmoid tumor in our case 1 was easily identified as a spiculated mass on FFDM as well as DBT, since the lesion was located in a fatty part of the breast (Fig. 1a and b). The small subtle desmoid presenting as distortion in case 2, however, was not diagnosed on synthesized 2D mammograms but clearly depicted on DBT (Fig. 2a-d). 
In conclusion, desmoid tumor is a rare benign lesion of the breast, which may present both clinically and radiographically as highly suspicious for malignancy. Tomosynthesis has the potential to detect the small subtle lesions of this aggressive fibromatosis manifesting as architectural distortion or spiculated mass that are occult on conventional mammography.

\section{Declaration of conflicting interests}

The author(s) declared no potential conflicts of interest with respect to the research, authorship, and/or publication of this article.

\section{Funding}

The author(s) received no financial support for the research, authorship, and/or publication of this article.

\section{References}

1. Benej R, Mečiarová I, Pohlodek K. Desmoid-type fibromatosis of the breast: a report of 2 cases. Oncol Lett 2017; 14:1433-1438.

2. Bhat D, Wear V, Weisenberg E, et al. Desmoid-type fibromatosis of the breast: a case report. Breast Dis 2016;36: 149-152.

3. Kelli YH, Patricia DL, Hamilton R. Breast fibromatosis mimicking breast carcinoma. Proc (Bayl Univ Med Cent) 2013;26:22-24.

4. Oh C, Hammoudeh ZS, Carlsen BT. Desmoid tumor following abdominally-based free flap breast reconstruction. Gland Surg 2017;6:89-92.

5. Choi KA, Yeong YA. Desmoid tumor of the chest wall mimicking recurrent breast cancer: multimodality imaging findings. Iran J Radiol 2016;13:e31649.

6. Erguvan-Dogan B, Dempsey PJ, Ayyar G, et al. Primary desmoid tumor (extraabdominal fibromatosis) of the breast. Am J Roentgenol 2005;185:488-489.

7. López-Ruiz J, Ruiz M, Echevarria JJ, et al. Mammary fibromatosis mimicking recurrent breast cancer: radiological findings. Eur Radiol 2005;15:2034-2036.

8. Eastley N, McCulloch T, Esler C, et al. Extra-abdominal desmoid fibromatosis: a review of management, current guidance and unanswered questions. Eur J Surg Oncol 2016;42:1071-1083.
9. Kasper B, Ströbel P, Hohenbergera P. Desmoid tumors: clinical features and treatment options for advanced disease. Oncologist 2011;16:682-693.

10. Lakhani SR, Ellis IO, Schnitt SJ, et al. WHO Classification of Tumours of the Breast, 4th ed. Lyon: IARC Press, 2012.

11. Rakha EA, Aleskandarany MA, Lee AHS, et al. An approach to the diagnosis of spindle lesions of the breast. Histopathology 2016;68:33-44.

12. Schnitt SJ, Collins LC. Biopsy Interpretation of the Breast, 2nd ed. Philadelphia, PA: Wolters Kluwer/ Lippincott Williams \& Wilkins, 2013.

13. Neuman HB, Brogi E, Ebrahim A, et al. Desmoid tumors (fibromatoses) of the breast: a 25-year experience. Ann Surg Oncol 2008;15:274-280.

14. Zavlin D, Ruff ES, Albright SB. Breast desmoid tumor after ductal carcinoma treatment: salvaging a DIEP flap reconstruction. Plast Reconstr Surg Glob Open 2016;4: e1142.

15. Matherne TH, Green A Jr, Tucker JA, et al. Fibromatosis: the breast cancer imitator. South Med J 2004;97:1100-1103.

16. Skaane P, Verhage CC, Westgard T, et al. Extra-abdominal desmoid tumor of the axilla. RoFo1980 132:349-351.

17. Vedantham S, Karellas A, Vijayaraghavan GR, et al. Digital breast tomosynthesis: state of the art. Radiology 2015;277:663-684.

18. Skaane P, Bandos AI, Gullien R, et al. Comparison of digital mammography alone and digital mammography plus tomosynthesis in a population-based screening program. Radiology 2013;267:47-56.

19. Partyka L, Lourenco AP, Mainiero MB. Detection of mammographically occult architectural distortion on digital breast tomosynthesis screening: initial clinical experience. Am J Roentgenol 2014;203:216-222.

20. Ray KM, Turner E, Sickles EA, et al. Suspicious findings at digital breast tomosynthesis occult to conventional mammography: imaging features and pathology findings. Breast J 2015;21:538-542.

21. Zuley ML, Guo B, Catullo VJ, et al. Comparison of twodimensional synthesized mammograms versus original digital mammograms alone and in combination with tomosynthesis images. Radiology 2014;271:664-671. 